\title{
Mineração
}

\section{Análise de múltiplas variáveis no fechamento de mina - Estudo de caso da pilha de estéril BF-4, Mina Osamu Utsumi, INB Caldas, Minas Gerais}

\author{
(Multiple account analysis in mine closure - A case study of the \\ waste dump BF-4, Osamu Utsumi Mine, INB Caldas, Minas \\ Gerais)
}

\author{
Flávia Andrade Nóbrega \\ PPGEM/DEMIN/UFOP.E-mail: faefei@yahoo.com.br \\ Hernani Mota de Lima \\ PPGEM/DEMIN/UFOP.E-mail:hernani.lima@ufop.br \\ Adilson do Lago Leite \\ DECIV/UFOP.E-mail: adilson@em.ufop.br
}

\section{Resumo}

A Mina Osamu Utsumi das Indústrias Nucleares do Brasil (INB), localizada no município de Caldas/MG esteve em operação de 1982 a 1995, quando ocorreu a paralisação definitiva da lavra e tratamento de minério de urânio. Desde então, a INB tem, como principal passivo, para fins de fechamento, a drenagem ácida gerada na cava, nas pilhas de estéril e na praia de rejeitos. A drenagem ácida de mina (DAM) na Mina Osamu Utsumi é relevante, tanto do ponto de vista ambiental, quanto pelos custos envolvidos na neutralização desta. Em dezembro de 2004, a INB assinou um termo de referência para elaboração e apresentação do plano de fechamento solicitado conjuntamente pelo IBAMA e CNEN. Diversos estudos têm sido realizados como subsídio à preparação do plano de fechamento. Esse artigo apresenta a aplicação do método da Análise de Múltiplas Variáveis (AMV) no estudo da melhor alternativa para o fechamento do "Bota-Fora" BF-4 da INB, dado seu potencial de geração de DAM.

Palavras-chave: Análise de múltiplas variáveis, depósito de estéril, fechamento de mina.

\begin{abstract}
The Osamu Utsumi Mine of the Indústrias Nucleares do Brasil (INB), located in Caldas/MG, extracted and processed uranium from 1982 to 1995, after which, operations were suspended, leaving INB with the liability for mine closure, part of which includes problems with acid drainage from the pit, waste dumps and tailings beach. Acid mine drainage (AMD) at the Osamu Utsumi Mine is relevant because of its environmental impact and its neutralization cost. In December, 2004, INB signed an agreement to prepare and present a closure plan according to IBAMA and CNEN requirements. Since that time several studies are in place in order to subsidise the preparation of the closure plan. This paper presents the study of the best alternative for closing the "Bota-Fora" BF-4, due its $A M D$ generation potential, using the Multiple Account Analysis (MAA) methodology.
\end{abstract}

Keywords: Multiple account analysis, waste dump, mine closure. 
Análise de múltiplas variáveis no fechamento de mina - Estudo de caso da pilha de estéril BF-4...

\section{Introdução}

O Complexo Mínero-Industrial de Poços de Caldas (CIPC), operou de 1982 a 1995 as atividades de lavra, beneficiamento e tratamento químico do minério de urânio, provenientes da Mina Osamu Utsumi, constituindo no primeiro complexo mínero-industrial para a produção de concentrado de urânio no Brasil. Com a exaustão das reservas da jazida de Caldas e da descoberta das reservas de Caetité, encontra-se em fase de descomissionamento e fechamento. Em fevereiro de 2004, o IBAMA, juntamente com a Comissão Nacional de Energia Nuclear (CNEN), firmou um Termo de Referência para Elaboração e Apresentação do Plano de Recuperação de Áreas Degradadas (PRAD), para o CIPC (Flôres, 2006).

O principal passivo enfrentado pela INB-Caldas, para o fechamento do CIPC, é a drenagem ácida de mina (DAM). A DAM é causada, basicamente, pela oxidação natural dos sulfetos metálicos (expostos na cava, pilha de estéreis e barragem de rejeitos) em presença de água e oxigênio. Como resultado, há formação de ácido sulfúrico, redução do pH e dissolução de metais pesados associados às matrizes rochosas com graves impactos ambientais. O BF-4, um dos depósitos de estéril da Mina Osamu Utsumi tem uma importante contribuição na geração de DAM no CIPC.
Esse artigo apresenta os resultados obtidos da adoção do Método de Análise de Múltiplas Variáveis (AMV) na seleção da melhor alternativa para o fechamento do Bota-Fora BF-4.

\section{Estudo de caso: Bota-Fora BF - 4}

Os estéreis da mina Osamu Utsumi são constituídos pelo material de decapeamento da jazida e pela rocha estéril da mina resultante da triagem nas frentes de lavra. Ao longo da vida da mina, foram gerados $45 \times 10^{6} \mathrm{~m}^{3}$ de estéreis (GEOTECH, 2005). O Bota-Fora BF-4 possui uma superfície de $569 \times 10^{3} \mathrm{~m}^{2} \mathrm{e}$ umvolume de estéril depositado de $12,4 \times 10^{6} \mathrm{~m}^{3}$, no vale do Córrego da Consulta, pertencente à bacia do Rio Verde, em área contígua a cava da mina. A Figura 1 apresenta duas vistas do BF-4. O processo construtivo do BF-4 ocorreu na forma de "ponta de aterro", gerando segregação granulométrica (frações mais grosseiras na base do BF-4 e as frações mais finas na parte superior deste). Esse tipo de disposição resulta em maior permeabilidade e capacidade de armazenamento de água e favorecimento da geração de DAM.

A Tabela 1 apresenta parâmetros da qualidade das águas de drenagem do BF-4, segundo Fernandes e Franklin (2000) e Souza et al. (1995), bem como os valores permitidos por lei, referentes à Portaria $n^{\circ}$ 518/04 do Ministério da Saúde (padrão de potabilidade) e à Resolução $n^{0}$ 357/05 do CONAMA (critérios de classificação de uso). O BF-4, também, configura-se como a fonte de maior contribuição no incremento de atividade de $\mathrm{U}_{238}$ e $\mathrm{Ra}_{226}$ nas águas do ribeirão Soberbo (Amaral, 1992), conforme demonstrado na Tabela 2.

No início das operações do CIPC, as drenagens ácidas do BF-4 eram misturadas com água da mina e lançadas no córrego do Consulta (Cipriani, 2002). Dados de monitoramento de água no local indicaram um aumento no teor de urânio e elementos estáveis nas águas de drenagem dos "bota-foras” e da cava da mina e evidenciaram a necessidade de implantação de um sistema de coleta, bombeamento e posterior tratamento ativo, segundo exigência da CNEN, que visava à neutralização da acidez, precipitação de radionuclídeos e metais pesados e decantação dos sólidos em suspensão (Cipriani, 2002; Fagundes, 2005).

\section{Tratamento ativo da drenagem ácida}

O tratamento ativo da DAM na INB Caldas consiste na adição de uma solução de cal hidratada, fazendo com que o $\mathrm{pH}$ - da ordem de 3,0 - se eleve até 10,0. A seguir, com o auxílio da adição de flocu-
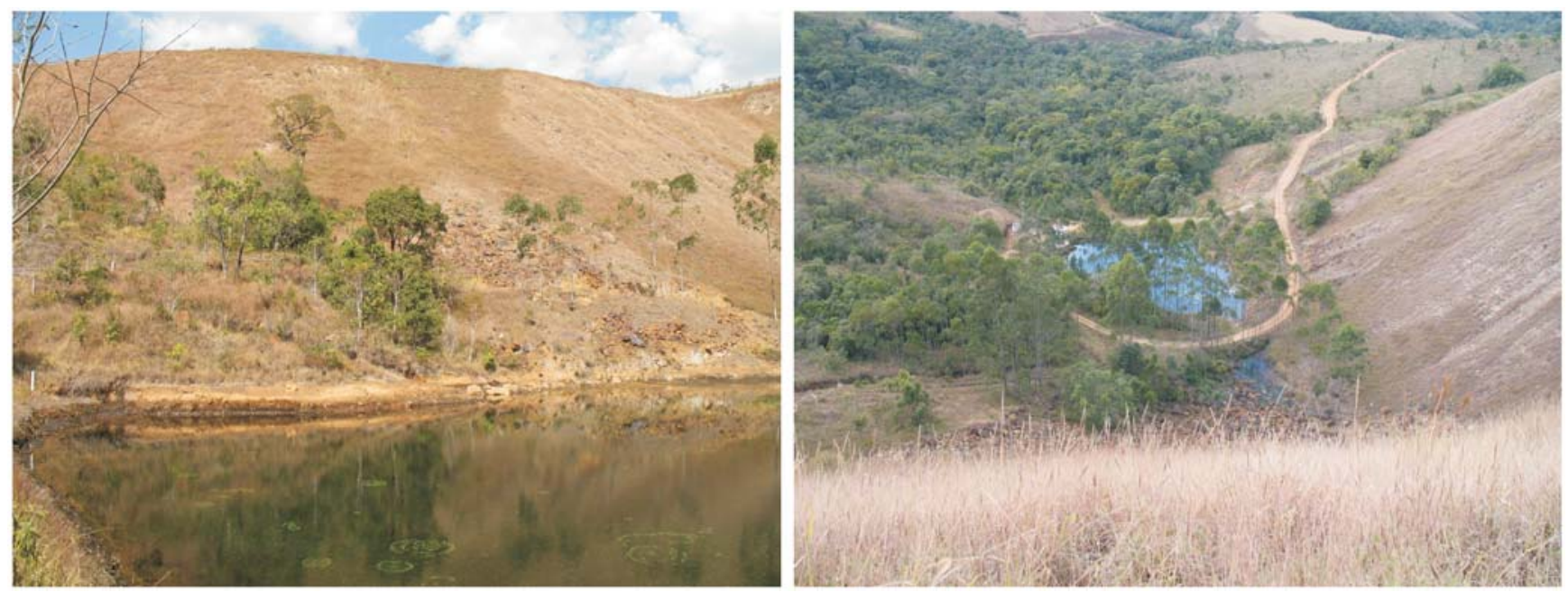

Figura 1 - Vista da base do BF-4 (esquerda) e vista do topo do BF-4 (direita) tendo ao fundo a bacia de captação da drenagem oriunda do BF-4. 
Flávia Andrade Nóbrega et al.

lantes, dão-se a precipitação e a decantação dos metais dissolvidos, antes que as águas retornem ao ambiente externo ao projeto. O volume médio de águas ácidas drenadas na base do BF-4 é de $157 \mathrm{~m}^{3} / \mathrm{h}$ (funcionando $12 \mathrm{~h}$ por dia). Esse volume é bombeado para a cava e, daí, juntamente com a água da cava, para a estação de tratamento. $\mathrm{O}$ volume de água diariamente tratado na estação é da ordem de $300 \mathrm{~m}^{3} / \mathrm{h}$.

O consumo de cal hidratada é da ordem de $1,40 \mathrm{~kg}$ de $\mathrm{cal} / \mathrm{m}^{3}$ de água. No ano de 2005, tratou-se um volume total de 2,1 x $10^{6} \mathrm{~m}^{3}$ de água, consumindo-se 2,9 t de cal hidratada (consumo médio da ordem de 200 t/mês) e 1,5 t de floculante (polieletrólito). O custo mensal do tratamento, considerando-se apenas o consumo desses dois insumos, variou entre $\mathrm{R} \$ 800.000,00$ e $\mathrm{R} \$ 900.000,00$.

O resultado final do tratamento é o extravasamento de uma água com $\mathrm{pH}$ médio da ordem de 10,0, que retorna ao ambiente, e a decantação de uma lama residual contendo, basicamente, manganês, alumínio, ferro, fluoretos e pequena porcentagem de urânio (aproximadamente $0,3 \%)$. Aágua tratada, com $\mathrm{pH}$ médio da ordem de 10,0, encontra outras águas fugidias acidificadas, no seu percurso em direção ao ambiente externo, de forma que o $\mathrm{pH}$ das águas descartadas, na interface entre os dois ambientes (o ambiente do complexo industrial e o ambiente externo) oscila entre 6,5 e 6,7 (Flôres, 2006).

\section{Metodologia da análise de múltiplas variáveis (AMV)}

O AMV é uma ferramenta de auxílio no processo de tomada de decisão. Através do $\mathrm{AMV}$, consideram-se os pontos positivos e negativos de cada alternativa por meio da identificação, quantificação, avaliação e comparação dos diversos elementos componentes das alternativas estudadas. As etapas desenvol-

Tabela 1 - Concentrações de elementos químicos das águas de drenagem do BF-4.

\begin{tabular}{c|c|c|c}
\hline $\begin{array}{c}\text { Espécie } \\
\text { Química }\end{array}$ & $\begin{array}{c}\text { BF-4 } \\
(\mathbf{m g} / \mathbf{l})\end{array}$ & $\begin{array}{c}\text { Portaria } \\
\mathbf{n}^{\circ} \mathbf{5 1 8 / 0 4}(\mathbf{m g} / \mathbf{l})\end{array}$ & $\begin{array}{c}\text { Resolução } \\
\mathbf{n}^{\circ} \mathbf{3 5 7 / 0 5}(\mathbf{m g} / \mathbf{l})^{*}\end{array}$ \\
\hline $\mathrm{Al}$ & 118 & 0,2 & 0,2 \\
\hline $\mathrm{SO}_{4}$ & 1010 & 250 & 250 \\
\hline $\mathrm{Mn}$ & 100 & 0,1 & 0,5 \\
\hline $\mathrm{F}$ & 100 & 1,5 & 1,4 \\
\hline $\mathrm{pH}$ & 3,3 & $6 \mathrm{a} 9,5$ & 6 a 9
\end{tabular}

Fonte: Modificado de Fagundes (2005).

${ }^{*}$ ) valores estabelecidos para as águas de classe 3.

Tabela 2 - Concentrações de elementos radioativos encontrados nas águas de base do BF-4.

\begin{tabular}{c|c|c|c|c|c}
\hline Pontos & ${ }^{232} \mathrm{Th}(\mathrm{Bq} / \mathrm{I})$ & ${ }^{238} \mathrm{U}(\mathrm{Bq} / \mathrm{I})$ & ${ }^{210} \mathrm{~Pb}(\mathrm{~Bq} / \mathrm{l})$ & ${ }^{226} \mathbf{R a}(\mathrm{Bq} / \mathrm{I})$ & ${ }^{228} \mathbf{R a}(\mathrm{Bq} / \mathrm{I})$ \\
\hline $\begin{array}{c}\text { Saída do } \\
\text { BF4* }\end{array}$ & 1,26 & 133,4 & 0,705 & 0,34 & 0,34 \\
\hline $\begin{array}{c}\text { Limites de } \\
\text { lançamento } \\
\text { CNEN }\end{array}$ & 1 & 1 & 1 & 1 & 1 \\
\hline
\end{tabular}

Fonte: Modificado de GEOTCH (2005), (*) dados referentes a agosto de 2005.

vidas para a realização dessa análise incluíram:

1. Adaptação do método às condições locais.

2. Trabalho de campo para coleta de dados e entrevistas com funcionários da INB.

3. Seleção de alternativas para o fechamento do BF-4.

4. Montagem de um banco de dados

5. Proposição de pesos das variáveis e indicadores e valores dos indicadores.

6. Emprego do Método de Análise de Múltiplas Variáveis para as alternativas listadas.

7. Análise dos resultados.

As alternativas analisadas foram selecionadas baseando-se no PRAD proposto pela INB.
Alternativa 1 - Trata-se de deixar o Bota-Fora 4 da forma como está, mantendo-se todas as atividades hoje realizadas, como, por exemplo, o bombeamento da drenagem para a cava e, posteriormente, para a estação de tratamento de ativo.

Alternativa 2 - Retaludar o BF-4, melhorar o sistema de drenagem superficial, bem como a colocar uma camada impermeabilizante para reduzir a infiltração de água e, conseqüentemente, minimizar a geração de drenagem ácida.

Alternativa 3 - Remover o BF-4 pela redisposição do estéril na cava.

O primeiro passo do processo de análise das variáveis relacionadas às alternativas para o fechamento do BF-4 foi a criação de um banco de dados. Este incluiu listagem das variáveis dos dife- 
Análise de múltiplas variáveis no fechamento de mina - Estudo de caso da pilha de estéril BF-4...

rentes impactos de cada alternativa. Em seguida, determinaram-se os indicadores especificados para cada variável, que descrevem cada impacto (Shaw et al., 2001). Quatro variáveis foram selecionadas: a técnica, a ambiental, a econômica e a social, de modo a abranger os possíveis impactos das alternativas consideradas para o plano de fechamento do Bota-Fora BF-4.

O procedimento de decisão de valores de base foi o segundo passo do processo de análise e envolveu "ranqueamento”, escalonamento e adição de peso (Robertson \& Shaw, 2004). O terceiro passo refere-se à determinação de valores. Os pesos das variáveis foram dados de acordo com a importância destas, segundo julgamento do avaliador. Os indicadores receberam pesos independentes das alternativas, considerando-se, apenas, a importância de cada fator. Os pesos das variáveis e indicadores variam de 1 a 5 , onde 1 corresponde ao menor peso e 5, ao maior. Cada indicador recebeu valores, considerando-se as variáveis técnica, ambiental, econômica e social. Os valores variaram de 1 a 9, pior a melhor, respectivamente.

A tabela de Análise de Múltiplas Variáveis (Tabela 3) apresenta as variáveis e os indicadores selecionados para a avaliação do processo e escolha da melhor alternativa para o BF-4, visando ao fechamento da Mina Osamu Utsumi. A aplicação da tabela e a valoração desta incluíram professores, pesquisadores e funcionários da INB convidados a participar do processo.

\section{Cálculos e resultados}

Utilizando-se os valores encontrados na Tabela 3, realizou-se o somatório de pontos de cada alternativa (Tabela 4), conforme demonstrado a seguir, seguindo-se a comparação das alternativas.
Tabela 3 - Dados quantitativos da análise da melhor alternativa.

\begin{tabular}{|c|c|c|c|c|c|c|}
\hline Variáveis & Peso & Indicadores & Peso & Alt. 1 & Alt. 2 & Alt. 3 \\
\hline \multirow{7}{*}{ Técnica } & \multirow{7}{*}{5} & Estética & 1 & 1 & 4 & 9 \\
\hline & & Estabilidade & 4 & 6 & 7 & 9 \\
\hline & & Erodibilidade & 4 & 6 & 7 & 8 \\
\hline & & Drenagem & 5 & 4 & 9 & 8 \\
\hline & & Bombeamento & 5 & 9 & 6 & 9 \\
\hline & & Radiação & 1 & 8 & 8 & 8 \\
\hline & & Geração de DAM & 5 & 1 & 8 & 9 \\
\hline \multirow{6}{*}{ Ambiental } & \multirow{6}{*}{5} & Estética & 4 & 1 & 6 & 9 \\
\hline & & $\begin{array}{l}\text { Recuperação da } \\
\text { vegetação }\end{array}$ & 5 & 2 & 6 & 7 \\
\hline & & $\begin{array}{l}\text { Qualidade da água } \\
\text { superficial }\end{array}$ & 5 & 1 & 8 & 9 \\
\hline & & $\begin{array}{l}\text { Qualidade da água } \\
\text { subterrânea }\end{array}$ & 5 & 2 & 6 & 9 \\
\hline & & $\begin{array}{l}\text { Restabelecimento } \\
\text { biológico }\end{array}$ & 4 & 1 & 7 & 7 \\
\hline & & Geração de DAM & 5 & 1 & 8 & 9 \\
\hline \multirow{5}{*}{ Econômica } & \multirow{5}{*}{4} & $\begin{array}{l}\text { Custos de } \\
\text { recuperação }\end{array}$ & 5 & 9 & 2 & 1 \\
\hline & & Custos (VPL) & 4 & 9 & 2 & 1 \\
\hline & & $\begin{array}{l}\text { Custos de } \\
\text { tratamento da água } \\
\text { (longo prazo) }\end{array}$ & 5 & 1 & 9 & 9 \\
\hline & & $\begin{array}{l}\text { Custos de } \\
\text { tratamento da água } \\
\text { (curto prazo) }\end{array}$ & 1 & 3 & 7 & 7 \\
\hline & & Geração de DAM & 5 & 1 & 7 & 9 \\
\hline \multirow{7}{*}{$\begin{array}{c}\text { Sócio - } \\
\text { econômica }\end{array}$} & \multirow{7}{*}{2} & Estética & 2 & 1 & 3 & 5 \\
\hline & & Recreação & 2 & 1 & 1 & 1 \\
\hline & & Turismo & 3 & 1 & 1 & 1 \\
\hline & & Saúde e bem-estar & 5 & 1 & 8 & 9 \\
\hline & & Infra-estrutura & 4 & 4 & 4 & 4 \\
\hline & & Empregos & 5 & 6 & 6 & 6 \\
\hline & & Geração de DAM & 5 & 1 & 8 & 9 \\
\hline
\end{tabular}


Para cada uma das variáveis de cada alternativa:

Variável $=\frac{\sum(\text { pesos dos indicadores } \times \text { valor escalar })}{\sum(\text { pesos dos indicadores })}$

Análise de múltiplas $=\frac{\left.\sum \text { (pesos das variáveis } \times \text { variáveis }\right)}{\sum(p e s \text { das varáveis) }}$

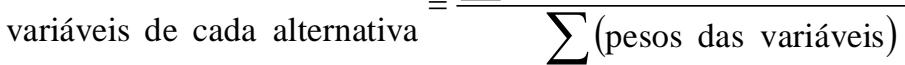

\section{Análise de custos}

O potencial de geração de drenagem ácida do BF-4, os custos de bombeamento da água ácida da BNF, para a cava da mina e, posteriormente, para a Unidade de Tratamento de Águas Marginais (AA-440), bem como os custos de tratamento da DAM, foram considerados para essa análise.

Para a Alternativa 1, usou-se o custo de R\$ 12.000.000,00 anuais, segundo dados da INB-Caldas. Nesse caso, há a necessidade de um tratamento constante, o que implica custo alto e de longo prazo. Para possibilitar uma comparação de custos

Tabela 4 - Valores da AMV para cada alternativa selecionada.

\begin{tabular}{c|c|c|c}
\hline Variável & Alternativa 1 & Alternativa 2 & Alternativa 3 \\
\hline Técnica & 5,29 & 7,32 & 8,60 \\
\hline Ambiental & 1,36 & 6,86 & 8,36 \\
\hline Econômica & 4,70 & 5,25 & 5,30 \\
\hline Sócio-econômica & 2,42 & 5,27 & 5,81 \\
\hline Resultado/alternativa & 11,50 & 19,76 & 22,62 \\
\hline
\end{tabular}

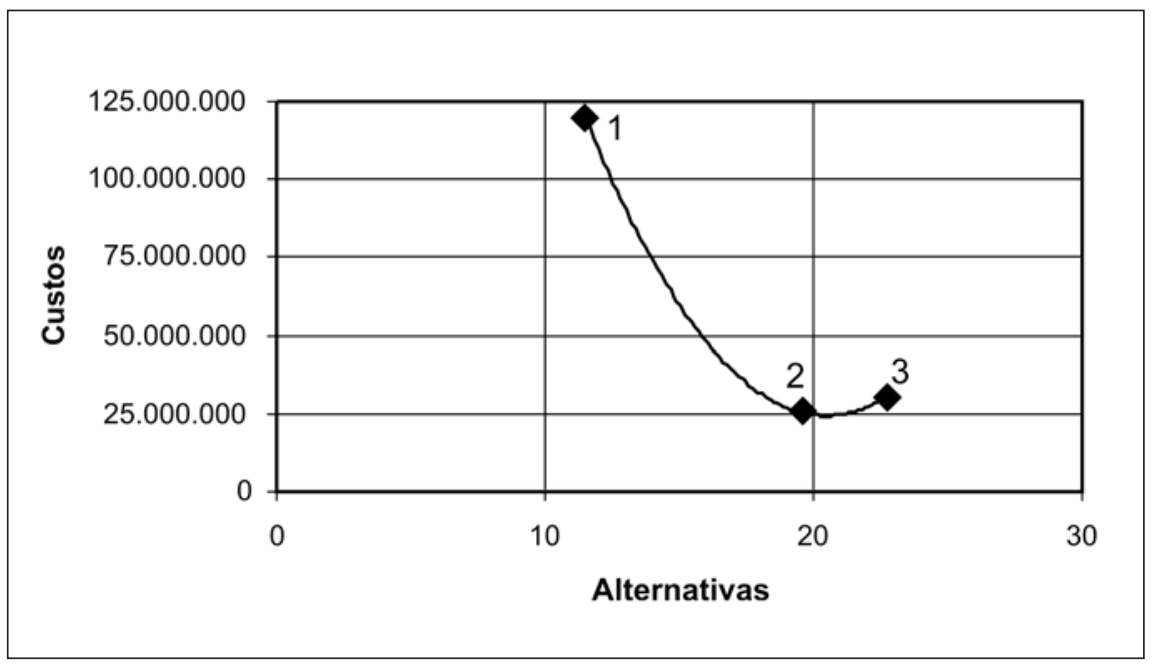

Gráfico 1 - Análise das alternativas x Custos. entre as alternativas propostas, considerou-se o custo relativo ao tempo estipulado para a conclusão das alternativas de retaludamento e transferência do BF-4 para a cava da mina, estimado em 10 anos. O PRAD apresentado pela INB considera, para a alternativa "recobrimento dos bota-foras com camadas impermeáveis”, um custo entre R\$ 25 e 50 milhões. Na AMV, para a Alternativa 2, adotou-se o valor de R $\$ 25$ milhões, pois o estudo se limita ao BF-4. Para a Alternativa 3, o PRAD considerou R \$ 50 milhões, sendo utilizado na AMV o valor de R \$ 30 milhões, considerando-se, apenas, o BF-4.

O Gráfico 1 mostra, no eixo das abscissas, os valores encontrados na Tabela 4 da análise de cada alternativa e, no eixo das ordenadas, os valores de custos em longo prazo adotados para cada uma delas. Os indicativos 1, 2 e 3 mencionados no gráfico referem-se, respectivamente, às alternativas 1,2 e 3 , do processo da AMV.

\section{Considerações finais}

O método de análise de múltiplas variáveis pode ser utilizado em diversos processos de tomada de decisão e, em especial, para o caso de fechamento de mina (ver, por exemplo, Shaw et al., 2001). Os atores envolvidos, bem como o caráter multidisciplinar, fornecem meios pelos quais os avaliadores podem selecionar a alternativa mais apropriada e vantajosa, através da ponderação relativa dos benefícios e custos de cada uma das alternativas possíveis.

No fechamento da Mina Osamu Utsumi, a drenagem ácida apresenta-se como o passivo de maior relevância, tanto do ponto de vista ambiental, quanto pelos custos envolvidos na neutralização desta. A dificuldade de comparação entre as possíveis alternativas para o fechamento da Mina Osamu Utsumi, com ênfase na drenagem ácida, foi uma das justificativas de se adotar o método de análise de múltiplas variáveis. 
Para a análise aplicada à INB-Caldas, buscou-se envolver o maior número possível de profissionais experientes que representassem diversas áreas do estudo, para garantir o sucesso e a confiabilidade dos resultados. A multidisiplinaridade, disposição, participação aberta e senso comum do grupo permitiram um resultado de consenso.

A melhor alternativa é aquela que obtiver maior pontuação. Portanto a alternativa de remoção do material estéril depositado no BF-4 e de disposição deste dentro da cava foi a que se apresentou como melhor opção. Geralmente a alternativa de maior pontuação é, também, a que gera maiores despesas. Mas através do Gráfico 1, pode-se avaliar que a pior alternativa, na AMV, é a que produz maiores despesas para a empresa. Portanto a adoção da remoção do estéril do BF-4 e a disposição na cava da mina representam uma solução economicamente e ambientalmente atrativa em longo prazo.

\section{Referências bibliogáficas}

AMARAL, E. Modificação da exposição à radiação natural devido a atividades agrícolas $e$ industriais numa área de radioatividade natural elevada no Brasil. UFRJ: 1992. (Tese).

BRASIL. Portaria n 518 do Ministério da Saúde, de 25 de março de 2004. Disponível em: http:// portal.saude.gov.br/portal/arquivos/ pdf/portaria_518_2004.pdf.

BRASIL. Resolução n ${ }^{0} 357$ do Conselho Nacional do Meio Ambiente, de 17 de março de 2005 - Classificação dos corpos de água. Ministério do Meio Ambiente. Brasil. 2005. 23 p.

CIPRIANI, M. Mitigação dos impactos sociais e ambientais decorrentes do fechamento definitivo de minas de urânio. Campinas: UNICAMP, 2002. (Tese).

FAGUNDES, J.R.T. Balanço hídrico do bota-fora BF4 da Mina Osamu Utsumi, INB, como subsídio para projetos de remediação de drenagem ácida. Ouro Preto: UFOP, 2005. (Dissertação).

FERNANDES, H. M., FRANKLIN, M. R. Assessment of acid rock drainage pollutants release in the uranium mining site of Poços de Caldas Brazil. Journal of Environmental Radioactivity, v. 54, 2000, p. 5-25.

FLÔRES, J. C. C. Fechamento de mina: aspectos técnicos, jurídicos e socioambientais. Campinas: UNICAMP, 2006. (Tese).
GEOTCH, CONSÓRCIO. Relatório técnico. Plano de recuperação de áreas degradadas. PRAD-UTMCaldas. R1. Dez., 2005.

ROBERTSON, A. M., SHAW, S.C. Use of the Multiple Accounts Analysis Process for Sustainability Optimization. In: SME Annual Meeting, Denver, Colorado, Feb 2325, 2004.

SHAW, S. C., ROBERTSON, A. M., MAEHL, W.C., KUIPERS, J., HAIGHT, S. Review of the Multiple Accounts Analysis Alternatives Evaluation Process Completed for the Reclamation of the Zortman and Landusky Mine Sites. National Association of Abandoned Mine Lands Annual Conference, Athens, Ohio, Aug 19-22, 2001.

SOUZA, V. P. Drenagens ácidas do estéril piritoso da Mina de Urânio de Poços de Caldas: interpretação e implicações ambientais. USP: 1996. 143 p. (Dissertação de Mestrado).

SOUZA et al. Gerenciamento de rejeitos da lavra de minério de urânio do CIP. In: SIMPÓSIOSOBREBARRAGENS DE REJEITOS E DISPOSIÇÃO DE RESÍDUOS - REGEO’ 95, 3.1995.

Artigo recebido em 18/06/2007 e aprovado em 15/02/2008.

\section{$\star * * * * *$}

\section{REM - Revista Escola de Minas 72 anos divulgando CIÊNCIA.}

$* * * * * *$

www.rem.com.br

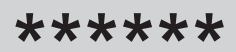

